\title{
Identifying a Need for Mental Health Services Posthospitalization in Adolescent Trauma Patients
}

Alexandra Hochstetler ${ }^{1}$, Ashley Vetor ${ }^{2}$, Jodi Raymond ${ }^{3}$, Hannah Bozell ${ }^{1}$, Teresa Bell ${ }^{2}$

${ }^{1}$ Indiana University School of Medicine; ${ }^{2}$ Indiana University School of Medicine, Department of Surgery; ${ }^{3}$ Riley Hospital for Children at IU Health

Background: Nearly 150,000 children were hospitalized due to an injury in 2018. Hospitalized patients are often prescribed opioids and as a result, one in eight adolescents will continue using opioids twelve months after hospitalization. Predictors of sustained opioid use and future misuse posthospitalization have yet to be studied in adolescents. One of these predictors may be mental health disorders following hospitalization. It is known mental health disorders can lead to substance use disorders if not addressed properly. In this study we examined the associations between injury severity, mental health, and substance use among adolescents.

Methods: Patients between 12 to 18 years old admitted for trauma were surveyed upon enrollment, and subsequently at 1, 3,6, and 12 months posthospitalization. These surveys measured anxiety, depression, posttraumatic stress, prescription and non-prescription drug use, pain severity and pain interference.

Results: At enrollment and one-month posthospitalization, higher pain interference was associated with anxiety $(p=.003)$, depression $(p<.001)$, and PTSD $(p=.004)$. Increased pain severity was also associated with higher PTSD $(p=.003)$ However, at three months, pain severity and interference were only associated with PTSD $(p=.005, p=.009)$. Frequent alcohol use and higher PTSD were found to be statistically significant at six and twelve months $(p=.02)$. Regular prescription opioid use was related to higher anxiety $(p=.048)$ and depression $(p=.048)$ only at enrollment and higher PTSD only at one month $(p=.034)$. Prescription opioid use was not associated with pain severity and interference at enrollment but was found significant at one month $(p=.016, p=.36)$.

Conclusion: Adolescents who reported higher pain severity and interference also more commonly had mental health disorders such as PTSD, anxiety, and depression. Higher PTSD scores and frequent alcohol use were also related post-injury. Screening for mental health after hospitalization should be further investigated in identifying adolescents who may be at risk for future opioid use disorders. 